RESEARCH REPORT

\title{
CORRELATION BETWEEN HIP ROTATION DYSFUNCTION AND KNEE PAIN
}

\begin{abstract}
BACKGROUND AND AIM

Hip and knee OA has been ranked as eleventh maximum contributor to global incapacity. The prevalence is expected to increase up to $25.9 \%$ by 2040 . Hip arthritis reduces the mobility at the hip joint limiting flexion extension, abduction, and adduction, internal and external rotation. Similarly knee pain causes considerable disability among middle adult population affecting both proximal and distal joint (hip and ankle) respectively. Hence the present study aims to find out a correlation between Hip Rotation Dysfunction and Knee Pain
\end{abstract}

\section{METHODOLOGY}

A cross sectional study was conducted on patient with hip and knee pain of age in between 45 to 60 years. Ranges at the level of hip joints were assessed using goniometer. Knee pain was assessed using Visual Analog Scale.

\section{RESULT}

The result obtained from the study shows a moderate negative correlation between knee pain and hip internal rotation, $r=-0.67$ showing no significant correlation $p=0.45$. There is also a weak negative correlation between knee pain and hip external rotation, $r=-0.37$ with a $p>0.05$.

\section{CONCLUSION}

The result obtained from the study concluded that no significant co-relationship exist between hip rotation dysfunction and knee pain, however more researches with larger number of sample size is required to further evaluate the causal relationship between the two variables.

\section{KEYWORDS}

Hip rotation, Correlation, dysfunction, knee pain, VAS, Prevalence.

\author{
Batool Hassan \\ Physiotherapist \\ Sindh Govt. Hospital \\ Hassan.batool.2016@gmail.com
}

\section{Anum Haidar}

Physiotherapist

Memon Medical Centre

anumladak@hotmail.com

\section{Nida Kanwal}

Physiotherapist

Maa Ayesha Clinic

Nida.kanwal@live.com

\section{Mehak Yaseen}

Physiotherapist

Chiniot General Hospital

[Hassan B, Haidar A, Kanwal N, Yaseen $M$. Correlation between Hip Rotation Dysfunction and Knee Pain. Pak. j. rehabil. 2017;6(2):32-37] 


\section{INTRODUCTION}

Hip and knee OA has been ranked as eleventh maximum contributor to global incapacity and 38th maximum in incapacity Adjusted Life Years out of 291 conditions globally. Prevalence is seen higher in females than guys. Handicap for hip and knee extended from 10.5 million to 17.5 Million from 1990 to $2010^{\prime}$. A research has been conducted in 2011 which revealed that around the world, osteoarthritis $(\mathrm{OA})$ is presently evaluated to be the fourth driving reason for inability. The greater part of this inability trouble is inferable from the contribution of the hips or the knees ${ }^{2}$

An expected 52.5 million (22.7\%) grown-ups in the United States have joint inflammation. Of those, 22.7 million (9.8\% of U.S. grown-ups) have joint inflammation inferable action impediment according to Morbidity and Mortality Weekly Report 2016. The prevalence of arthritis is projected to increase 78.4 million (25.9\% of U.S. adults) by 2040 , and the number of adults with AAAL is projected to increase to 34.6 million (11.4\% of U.S. adults).

Knee and hip osteoarthritis are profoundly common infections in the more established population. The estimation for hip substitution is by all accounts altogether higher than that for knee substitution. Out of 11,002 individuals, the assessed suitability rate for hip substitution seems $37.7 \%$ in men and $52.7 \%$ in women with osteoarthritis. The evaluated propriety rate for knee substitution seems $11.8 \%$ in men and $17.9 \%$ in women with osteoarthritis ${ }^{3}$.

Degenerative deformities at the joint leads to structural and functional limitations at the joint limiting its physiological movements.

Radiographic signs of Femoral acetabular impingement in asymptomatic adults with age in between 40 and 60 years shows increase in alpha angle is related to decrease internal rotation of hip joint leading to painful hip joint which may affect it's proximal and distal joint (Sacroiliac and Knee) respectively ${ }^{4}$.

According to the American Academy of Orthopedic Surgeons knee pain is a common condition resulting in 19.4 million pain-related visits to a physician each year. This number is significantly higher than low back pain (5.9 million visits), hip pain (3.2 million visits), and ankle pain (1.9 million visits). Knee pain impairs function, which can lead to disability 5 . This condition is profoundly pervasive in more seasoned people and is viewed as an essential determinant of general wellbeing status in maturity ${ }^{6}$. Agony in weight bearing joints might be because of various causes, including alluded or emanated torment, sciatica, delicate tissue malady. In any case, confirm is developing that personal satisfaction and incapacity in $\mathrm{OA}$ are more identified with indications than to radiological seriousness ${ }^{7}$. Hip arthritis reduces the mobility at the hip joint limiting flexion extension, abduction, and adduction, internal and external rotation. Similarly knee pain causes considerable disability among middle adult population affecting both proximal and distal joint (hip and ankle) respectively. Patients with osteoarthritis are at higher danger of death contrasted and the all-inclusive community. History of diabetes, tumor, or cardiovascular infection and the nearness of strolling incapacity are real hazard factors. Administration of patients with osteoarthritis and strolling inability should concentrate on compelling treatment of cardiovascular hazard variables and comorbidities, and also on expanding physical action ${ }^{8}$.

If we need to examine a patient with complain of pain in knee joint, it is not completed without examining adjacent joint, hip and ankle, respectively $y^{9}$.There is a connection between power and position of hip joint and Patellofemoral Joint Stress (PFJS) ${ }^{10}$. It is revealed by a study that strengthening of hip abductor muscles was very much productive in mounting muscle power and diminishing pain in patients with Patellofemaoral Pain Syndrome (PFPS) ${ }^{11}$.

The problem in the proximal (hip) joint can affect distal (tibiofemoral and patellofemoral) joint mechanics ${ }^{12}$. Hip strength is very much significant. It is concluded that any movement at hip joint whether flexion, extension, rotation, poor motor control at the hip often manifests itself in knee pain ${ }^{13}$. Severe knee osteoarthritis is a strong cause of hip and knee pain $^{14}$.

Loose or weak pelvis may contribute to knee injury. A strong upper leg strength can be beneficial in preventing knee injury ${ }^{15}$. The result of a study suggested that there is a strong relation between a weak hip and knee pain thus indicating a strong hip joint has a positive impact on distal knee joint and a weak hip joint may contribute to weaken the knee joint ${ }^{16}$. Periodic dislocation is seen in quite a few patients which give may arise to knee complications ${ }^{17}$. So the knee pain may have a direct or indirect relation with faulty biomechanics at hip and ankle joint ${ }^{18}$.

\section{METHODOLOGY}

\section{Participants}

Patients between age 45 to 60 years including both male and female.

\section{Research Design}

Cross sectional study design.

\section{Study Setting}

The research was conducted in Out Patient Depart- 
ment of Jinnah Hospital, Alamgir Health Care Center, Memon Medical Institute, Sindh Government Hospital.

\section{Study Duration}

6-8 months.

\section{Sample Size}

Sample size was calculated using WHO software for sample size calculation. The study conducted in 2010 title as "epidemiology of osteoarthritis" was taken to calculate the sample size. By taking the prevalence of hip OA $27 \%$, keeping confidence Interval $95 \%$ and $8 \%$ bound of error; a sample size of 115 was calculated.

\section{Sampling Method}

Subjects were allocated to study groups using convenient sampling method.

\section{Inclusion Criteria}

- The vast majority of the consideration criteria utilized were the understanding, capacity and readiness to partake in the outpatient technique ${ }^{19}$.

- Ranging from 45 to 80 years ${ }^{20}$.

- Patients with both hip and knee pain

- Not taking any drug during the inclusion period which could perturb walking (for example neuroleptics)

- No limitation for strolling or light physical activity

\section{Exclusion Criteria}

- Sign and symptoms of depression

- Any trauma and history of fall

- Grade 3 obese patients with BMI (>40kg/m2) will be excluded ${ }^{21}$

- Patients with cardiovascular (e.g. heart disappointment, history of myocardial dead tissue, arrhythmia), pneumonic (e.g. embolism, respiratory disappointment) and inadequately controlled comorbidities will be prohibited ${ }^{22}$.

- Systemic problems

- Open wounds

- External fixators

\section{Data collection procedure}

All the subjects were recruited with knee pain; detailed hip assessment by a senior therapist was done. All ranges were assessed using goniometer and pain on the knee will be assessed using VAS.

\section{Data Analysis}

Analysis was performed by the researcher using computer analysis software SPSS Version 21. Descriptive data for the average of the three measurements VAS, IR and ER included means and standard deviations. The Pearson's correlation coefficient was used to analyze the associations between hip and knee and to compare the pain of knee joint with hip rotation dysfunction. In order to adjust for possible inflation of the ( $P>0.05)$ alpha level due to multiple t-tests.

\section{Outcome Measures}

The visual analogue scale is a measurement tool which was used to assess intensity of pain which provides 0- 10 score in which higher score indicates high intensity of pain. VAS recommended score as no pain (0), mild pain (1 to 4), moderate pain (5 to 7) and severe pain (8 to 10). Another measurement instrument is goniometer which is used to measure ranges at hip joint.

\section{RESULT}

The study includes 100 participants with a mean age of 53.25. The male and female participants included in this study is shown in figure 1.

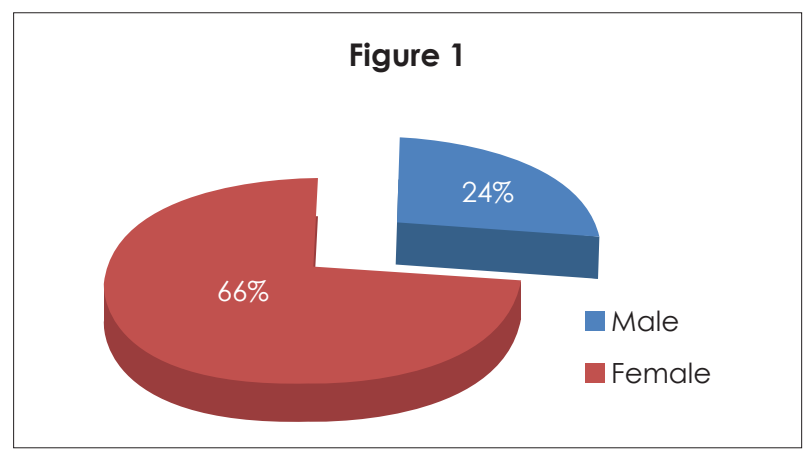

In the following table 1, the means and standard deviations of participants' age s shown and gender distribution of participant. There were in total 100 participants in this research study were included. The demographics show that there was mean age 50 years. There were $24 \%$ male and $66 \%$ female among participants.

Table1. Percentage distribution of demographic characteristics of knee pain patients

\begin{tabular}{|c|c|c|}
\hline $\begin{array}{c}\text { Demographic } \\
\text { characteristics }\end{array}$ & $\mathrm{n}$ & $\%$ \\
\hline Age (Mean \pm SD) years & $53.25 \pm 4.5$ & \\
\hline Gender & & \\
\hline Male & 24 & 24 \\
\hline Female & 66 & 66 \\
\hline
\end{tabular}

With pain scale 4 mean $I R=30.67$ and $E R=36$, at pain scale 5 mean $I R=32.88$ and $E R=34.52$, at pain level 6 mean $I R=27.77$ and $E R=30.06$, at 7 mean $I R=24.93$ and $E R=28.24$ and at pain scale 8 mean $I R=17.33$ and $E R=25$ was found as shown in Figure 2. 


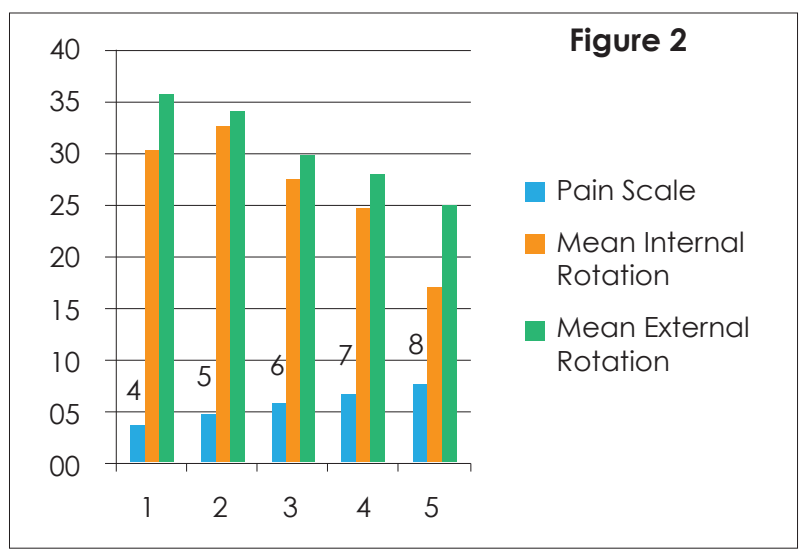

Mean value for pain scale was 6.40 with the average IR found to be 26.21 and ER 29.64 of the given sample with the SD 6.49 and 8.03 respectively as shown in Table 2.

\begin{tabular}{|c|c|c|}
\hline \multicolumn{3}{|c|}{$\begin{array}{c}\text { Table 2: Descriptive Statistics Of Pain Intensity (Vas), } \\
\text { Internal Rotation And External Rotation (N=100) }\end{array}$} \\
\hline Variable & Mean & Std. Deviation \\
\hline VAS & 6.40 & 1.03 \\
\hline IR & 26.21 & 6.49 \\
\hline ER & 29.64 & 6.49 \\
\hline
\end{tabular}

There is a moderate negative correlation between knee pain and hip internal rotation, $r=-0.67, p=0.45$. There is also a weak negative correlation between knee pain and hip external rotation, $r=-0.37, p=$ 0.13 .

\begin{tabular}{|c|c|c|}
\hline \multicolumn{3}{|c|}{$\begin{array}{c}\text { Table 3: Correlation Between Pain Intensity And } \\
\text { Internal Rotation, Pain Intensity And External Rotation } \\
(\mathbf{N}=100)\end{array}$} \\
\hline Variable & Pearson Correlation & P-Value \\
\hline VAS+IR & -0.674 & 0.45 \\
\hline VAS+ER & -0.373 & 0.13 \\
\hline
\end{tabular}

Correlation is significant at the 0.01 level (2-tailed).

\section{Graph 1}

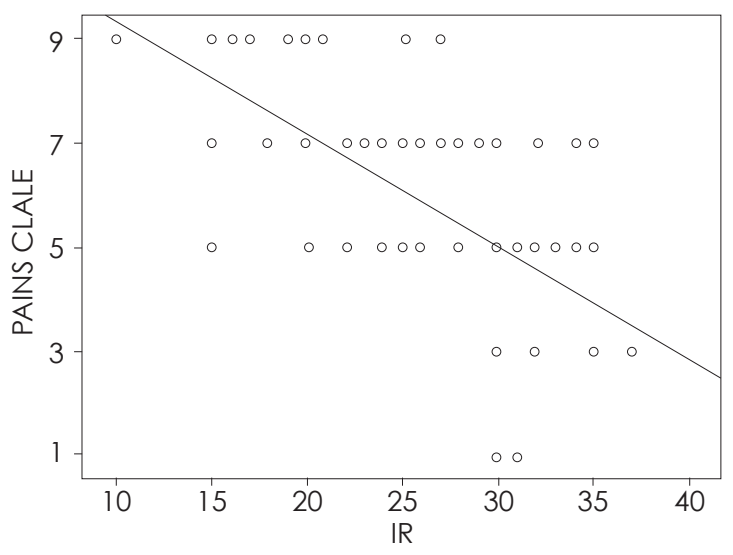

Graph 2

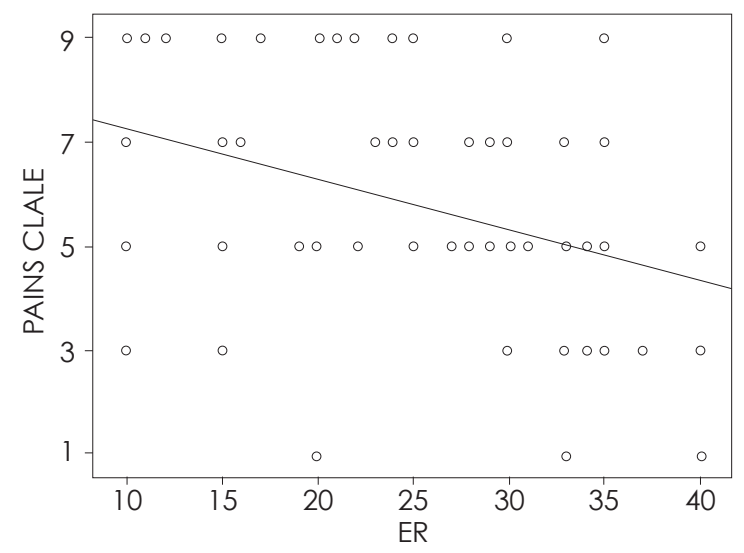

DISCUSSION

The purposes of this study were to determine whether there are differences in lower-extremity kinematics and to examine the relationship between hip Rotation and knee pain. Correlation between hip rotation dysfunction and knee pain has been checked on patients who have pain in knee for more than 3 months. Results shows that patients who have severe pain have decrease internal rotation at hip joint as compare to the patients who have moderate pain. External rotation is also reduced to some extent but we don't see any direct effect of knee joint pain on external rotation. As our interpretation of research there would be change in ranges depending on whether pain is moderate or severe.

There were no gender differences ( $P>0.05)$, which may be driven by the low subject numbers; however, due to the lack of gender differences, the correlational analyses were performed without regard to gender. Also, due to the moderate r-values reported for our significant findings, the results do not appear to be influenced by the low subject number.

Previous studies show a strong connection between hip and knee joint dysfunction that a faulty knee joint mechanics may influence hip joint function. According to Erik, When adduction or internal rotation increases at hip joint, it increases the $Q$ angle by increasing the relative valgus of the lower limb, thus pressure on the patellofemoral joint increases due to peal lateral contact ${ }^{23}$.

According to Gibson, pain and age of patient had inverse relation, younger patients report higher pain. Age difference in pain score have been reported but the cause of difference is not certain and questionable ${ }^{24}$. Herr believe that older adults are not familiar with pain, cultural factor like stoicism results in under reporting of pain in older adults. 
Suggest that cultural factors, such as stoicism, lack of familiarity with verbal pain descriptors and the practice of pain reporting, may result in under-reporting of pain among older adults ${ }^{25}$. Recently LaChapelle found that increasing age is related to decreased pain severity and the perception of greater control over pain. The literature suggests that under-reporting of pain by the older adult cohort may be commonplace and that older patients may perceive greater pain control and thus lower pain severity ${ }^{26}$.

For the future studies, check range and pain scale before and after treatment to see whether ranges are consistent or increases as pain decreases after the treatment. Age group of patients should be specific.

\section{CONCLUSION}

VAS for knee pain is not a reliable tool to extract the exact condition of knee joint pain at that particular time and unequal numbers of males and females and low number of subjects who participated in this investigation were the limitations of the study. To ensure the correlational analyses were not influenced by gender, separate one-way analyses of variance were performed to determine if there were significant differences between males and females for all variables of interest A final limitation of this investigation was the range of motion limits and positioning utilized for assessing Rotation of the hip joint.

\section{REFERENCES}

[1] Cross M, Smith E, Hoy D, Nolte S, Ackerman I, Fransen $M$ et al. The global burden of hip and knee osteoarthritis: estimates from the global burden of disease 2010 study. Ann Rheum Dis. 2014;73(7):1323-30

[2] Ganasegeran K, Menke JM, Ramaswamy C, Murthy V, Abdul Manaf R, Alabsi AM, Al-Dubai SA. Level and determinants of knowledge of symptomatic knee osteoarthritis among railway workers in Malaysia. BioMed research international. 2014

[3] Quintana JM, Arostegui I, Escobar A, Azkarate J, Goenaga JI, Lafuente I. Prevalence of knee and hip osteoarthritis and the appropriateness of joint replacement in an older population. Arch Intern Med. 2008;168(14):1576-84

[4] Diesel CV, Ribeiro TA, Scheidt RB, de Souza Macedo CA, Galia CR. The prevalence of femoroacetabular impingement in radiographs of asymptomatic subjects: a cross-sectional study. Hip Int. 2015;25:258-63

[5] Peat G, McCarney R, Croft P. Knee pain and osteoarthritis in older adults: a review of community burden and current use of primary health care. Ann Rheum Dis. 2001;60(2):91-7

[6] Dawson J, Linsell L, Zondervan K, Rose P, Randall T, Carr A, Fitzpatrick R. Epidemiology of hip and knee pain and its impact on overall health status in older adults. Rheumatology. 2004 ;43(4):497-504

[7] Salaffi F, Carotti M, Grassi W. Health-related quality of life in patients with hip or knee osteoarthritis: comparison of generic and disease-specific instruments. Clin rheumatol. 2005;24(1):29-37

[8] Nüesch E, Dieppe P, Reichenbach S, Williams S, Iff $S$, Jüni $P$. All cause and disease specific mortality in patients with knee or hip osteoarthritis: population based cohort study. BMJ. 2011;342:d1165

[9] Yilmaz AE, Atalar H, Tag T, Bilici M, Kara S. Knee joint pain may be an indicator for a hip joint problem in children: a case report. The Malaysian journal of medical sciences: Malays J Med Sci. $2011 ; 18(1): 79$

[10] Meira EP, Brumitt J. Influence of the hip on patients with patellofemoral pain syndrome: a systematic review. Sports Health. $2011 ; 3(5): 455-65$

[11] Ferber R, Kendall KD, Farr L. Changes in knee biomechanics after a hip-abductor strengthening protocol for runners with patellofemoral pain syndrome. J Athl Train. $2011 ; 46(2): 142-9$

[12] Powers CM. The influence of abnormal hip mechanics on knee injury: a biomechanical perspective. J Orthop Sports Phys Ther. 2010;40(2):42-51

[13] Van Dillen LR, Bloom NJ, Gombatto SP, Susco TM. Hip rotation range of motion in people with and without low back pain who participate in rotation-related sports. Phys Ther Sport. 2008;9(2):72-81.

[14] Zeni JA, Rudolph K, Higginson JS. Alterations in quadriceps and hamstrings coordination in persons with medial compartment knee osteoarthritis. J Electromyogr Kinesiol. 2010;20(1):148-54

[15] Bennell KL, Hunt MA, Wrigle TV, Hunter DJ, Hinman RS. The effects of hip muscle strengthening on knee load, pain, and function in people with knee osteoarthritis: a protocol for a randomized, single-blind controlled trial. BMC MusCuloskelet Disord. 2007;8:121

[16] Rowe J, Shafer L, Kelley K, West N, Dunning T, Smith $R$ et al. Hip strength and knee pain in females. North American journal of sports physical therapy: N Am J Sports Phys Ther. 2007;2(3):164-169

[17] Masaoka T, Yamamoto K, Shishido T, Katori $Y$, Mizove T, Shirasu H, Nunoda D. Study of hip joint dislocation after total hip arthroplasty. Int Orthop. 2006;30(1):26-30

[18] Cookson L. Atypical knee pain: the biomechanical and neurological relationship between the pelvis, hip and knee-a case report. Clin Chiropr. 2003;6(2):63-66 
[19] Aynardi M, Post Z, Ong A, Orozco F, Sukin DC. Outpatient surgery as a means of cost reduction in total hip arthroplasty: a case-control study. HSS J. 2014;10(3):252-5

[20] Berger RA, Kusuma SK, Sanders SA, Thill ES, Sporer $\mathrm{SM}$. The feasibility and perioperative complications of outpatient knee arthroplasty. Clin Orthop Relat Res. 2009;467(6):1443-9

[21] Berger RA, Sanders SA, Thill ES, Sporer SM, Della Valle C. Newer anesthesia and rehabilitation protocols enable outpatient hip replacement in selected patients. Clin Orthop Relat Res. 2009;467(6):1424-30

[22] Gondusky JS, Choi L, Khalaf N, Patel J, Barnett S, Gorab R. Day of surgery discharge after unicompartmental knee arthroplasty: an effective perioperative pathway. J Arthroplasty. 2014;29(3):516-519
[23] Hewett TE, Myer GD, Ford KR, Heidt Jr RS, Colosimo AJ, McLean SG et al. Biomechanical measures of neuromuscular control and valgus loading of the knee predict anterior cruciate ligament injury risk in female athletes: a prospective study. Am J Sports Med. 2005;33(4):492-501

[24] Gibson SJ, Helme RD. Age-related differences in pain perception and report. Clin Geriatr Med. 2001;17(3):433-56

[25] Herr KA, Mobily PR. Complexities of pain assessment in the elderly clinical considerations. J Gerontol Nurs. 1991;17(4):12-9.

[26] Roth $M L$, Tripp DA, Harrison $M H$, Sullivan $M$, Carson P. Demographic and psychosocial predictors of acute perioperative pain for total knee arthroplasty. Pain Res Manage. 2007;12(3):185-94. 